\title{
Genetically modified insects as used in SIT should not require regulation
}

\author{
Thomas Albert Miller
}

Received: 15 April 2011 / Accepted: 30 May 2011 /Published online: 22 June 2011

(C) Springer Science+Business Media B.V. 2011

Biography

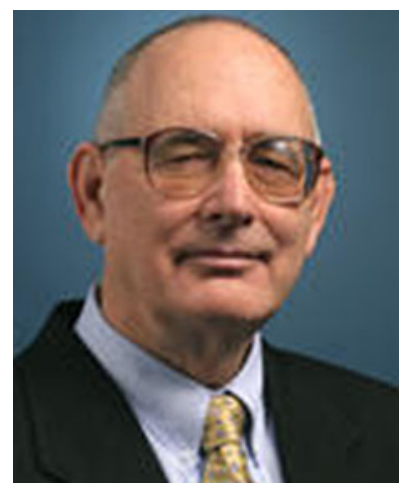

Thomas Albert Miller was born in Pennsylvania and moved to California, where he was educated and received a B.A. degree in physics (1962) and a Ph.D. in entomology (1967), both from the University of California, Riverside (UCR). In the graduate entomology program, Dr. Miller invented a transducer to record heartbeat of the American cockroach, Periplaneta americana and using this discovered the myogenic nature of the cockroach heartbeat. This was followed by pioneering neurophysiological methods to determine the function of cardiac neurons in the cockroach heart while a postdoctoral fellow at the

\section{T. A. Miller $(\bowtie)$}

Department of Entomology, University of California,

Riverside, CA 92521, USA

e-mail: thomas.miller@ucr.edu
University of Illinois and a NATO Postdoctoral Fellow at the University of Glasgow. Miller joined the staff of UCR in 1969 and for 15 years used neurophysiology to determine the mode of action of cyclodienes and later DDT and pyrethroid insecticides on houseflies, work supported by the National Institutes of Health. Because synthetic pyrethroids were a brand new chemistry, Dr. Miller wanted to document the development of resistance to them in field populations of cotton pests and started a program of research in support of the California cotton industry. He invented methods of measuring resistance to insecticides with a modified pheromone trap and beginning of diapause (dormancy) in pink bollworm with an antibody method, both taking $24 \mathrm{~h}$ and both applied in commercial cotton fields. Dr. Miller then developed a genetically modified pink bollworm for use in the Sterile Insect Technique (SIT) program. This took 10 years and led to the Gregor Mendel Gold Medal for research in the biological sciences, an award conferred by the Czech Academy of Sciences in 2003. While continuing an interest in SIT, Dr. Miller has helped develop the concept of symbiotic control with pioneering groups at Yale and elsewhere.

Dr. Miller was plenary speaker at the Plant Protection Congress, International Symbiosis Society Congress and International Congress of Insect Biotechnology and Industry. He founded the Springer Series of Experimental Entomology and Contemporary Topics in Entomological Research for Chapman \& Hall and later 
Taylor \& Francis publishing companies and serves on several editorial boards. He has been a consultant to dozens of agrochemical companies, the Wellcome Foundation, the US National Academy of Sciences, the Australia Cotton Growers, and the International Atomic Energy Agency. Dr. Miller has organized symposia at international scientific meetings in Canada, Korea, Turkey, Hungary and Vienna. Author or editor of 235 publications, including journal articles, books, chapters, reviews, proceedings and technical reports. $\mathrm{He}$ is a Fellow of the American Association for the Advancement of Science, the Entomological Society of America, the Royal Entomological Society, and was appointed a Jefferson Science Fellow by the U.S. Department of State in 2010.

In the fall of 2010, the government of the Cayman Islands made news by releasing male RIDL (Release of Insects carrying a Dominant Lethal gene) denguetransmitting mosquitoes, Aedes aegypti, developed by Oxitec, Ltd in the UK. An 80\% reduction in the native Ae. aegypti population was reported in the release area and followed a smaller preliminary release in 2009. Ae. aegypti transmits the dengue virus; given that there is no vaccine or specific drug, dengue control depends on control of this mosquito.

The tactic used for this transgenic (GM) version of the Sterile Insect Technique (SIT, Dyck et al. 2005) was to release only male mosquitoes (males do not take blood meals and therefore do not transmit the dengue virus). Females in the wild population that mate with RIDL male mosquitoes produce progeny unable to develop to the adult stage.

Applying this technique in the United States could make an important contribution toward curbing a dengue outbreak. Andrea Leal, Deputy Director of the Florida Keys Mosquito Control District, informed me that a dengue outbreak occurred there between May and October of 2009. Most likely a traveler brought dengue virus 1 back after acquiring it abroad and became the local source of infection. Twenty-seven cases were reported that year, and another 66 cases were reported from March to November of 2010.

In addition, individual, locally acquired cases were identified in Miami-Dade and Broward Counties. These two cases were caused by different dengue viruses and were unrelated to each other and to the virus transmission that occurred earlier in Key West. Leal mentioned that up to $50 \%$ of people infected with dengue viruses have unapparent infections, many of them being too mild to be recorded by a visit to medical facilities. A serological survey indicated that $5 \%$ of inhabitants of a sector of Key West have been infected (suggesting >1000 infections may have occurred in this area).

Dengue is widely reported to be spreading beyond traditional areas of infection. According to Gary Clark, at the USDA-ARS vector labs in Gainesville, Florida, the Key West outbreak in 2009 was the first evidence of local dengue transmission in the continental US outside of Texas in over 60 years. An infected person develops life-long immunity to a given strain (serotype) of the virus and soon loses the ability to act as an inoculation source; however, there is no cross-resistance to the three other serotypes, some of which are much more lethal than Dengue virus 1 (Popovici et al. 2010).

Insecticide-based methods for control of the dengue mosquito are effective to a degree, but are unable to eradicate the mosquito locally. Mosquito abatement personnel lack access to all breeding locations for a variety of reasons, the main one being absent owners with locked properties, which means that some dengue-infected mosquitoes always manage to avoid insecticide sprays. Also with decreasing population sizes, insecticide control methods decrease, whereas SIT methods increase in effectiveness.

Both GM and non-GM SIT methods are far more efficient than insecticides because the males actively seek out wild females including those in locations not accessible to control personnel. The public reaction to the news from non-governmental organizations (NGOs) and environmental groups was predictably vocal, if off-balanced (Enserink 2010). Complaints against use of GM mosquitoes in SIT are often poorly focused. See, for example, suggestions for improving the anti-GMO arguments from Mark Benedict (2011).

Instead, to allay the concerns of NGOs and environmental groups, a distinction can be made between GM insects used in SIT programs compared to other GM strategies. A group of students at the 2008 national meeting of the Entomological Society of America published results of a debate on use of genetically modified organisms in entomology (Nielsen and Burrus 2010). In the introduction, they determined that:

"For the purposes of this debate, GMOs included herbicide-resistant plants and organisms 
that are altered with Wolbachia, but did not include sterilized insects (Sterile Insect Technique). Sterilized insects were not considered GMOs because the sterilization conferred on an insect does not proceed to the next generation; the insects are incapable of reproducing the mutated genome."

With this very simple statement these young professionals have added clarity to this issue. A GM insect like the dengue mosquito described above that is used in the SIT sense is at a reproductive dead end.

SIT has been used successfully and safely for many years by governments and growers to protect local agricultural industries. The targets of these programs are various species of tsetse flies, fruit flies, moths and screwworm flies. Technical advice and technology development and transfer for many of these programs is provided primarily by the Insect Pest Control Subprogramme of the International Atomic Energy Agency, in partnership with the Food and Agricultural Organization, both part of the United Nations.

Sufficient doses of radiation to cause sterility unfortunately may cause damage to some tissues resulting in significant loss of competitiveness in sterile males. Radiation-based SIT requires ratios of SIT insects to wild types as high as 100:1 to block reproduction in field populations. Even with this level of inefficiency, SIT has been so successful at suppressing and in some cases even locally eradicating pest populations from large areas that it is now demanded by some trading partners to ensure pest-free produce.

While not necessary for successful SIT, a few programs lack an infallible marker system. In these cases SIT releases must continue due to inability to distinguish between SIT and wild type insects in monitoring traps after local populations are eliminated. In other programs DNA methods allowing 100\% identification have allayed grower fears that local outbreaks were caused by released insects.

As a biological control method, SIT is close to ideal. It involves only the pest insect itself. It does not involve importing parasites and predators from abroad; the pest is already present, so there are no invasive species or biodiversity issues. Moreover, the increase in efficiency by releasing only males in some cases means no blood meals (mosquitoes) and no fruit or vegetable damage (fruit flies, moths).
This would be arguably the most sustainable method of pest control in existence were it not for the need in some cases to reduce local populations first to manageable levels with insecticide, pheromone or other methods.

Use of GM insects in SIT, such as offered by the RIDL mosquito, would do several things: tests show that the RIDL mosquito is equally competitive or nearly so with non-GM mosquitoes (Gary Clark, USDA-ARS, Gainesville, FL, personal communication); genetic markers eliminate the identification problem, thereby removing uncertainty; and genetic methods of producing only males make that option available, although at present the yellow fever males are selected by hand because of a size difference with females.

The whole point of the Cartagena Protocol on Biosafety was to "... protect human health and the environment from the possible adverse effects of the products of modern biotechnology." Since RIDL mosquitoes and other GM insects used in SIT programs are at a reproductive dead end, there is no risk to assess and nothing to regulate.

All of the U.S. regulatory agencies reached a similar conclusion with the GM zebra fish and declined to regulate. As a result GloFish ${ }^{\circledR}$ is sold in 49 states including Virginia, where I live. GM insects used in SIT are designed to be biologically contained. At the end of operational release, no reproductive individuals remain. Apparently governments in Cayman Islands, Malaysia and Brazil reached similar conclusions in allowing RIDL trials.

Acknowledgments I thank Mark Benedict, Luke Alphey, Bob Rose, Jorge Hendrichs, and Gary ClarkKen Ferguson, for comments on the factual parts of early drafts. However, opinions expressed here are solely those of the author.

\section{References}

Benedict, M. Q. (2011). Happy New Year! Getting serious about GMO risks in 2011. http://www.malariaworld.org/ blog/happy-new-year-getting-serious-about-gmo-risks2011.

Dyck, V. A., Hendrichs, J., \& Robinson, A. S. (Eds.). (2005). Sterile insect technique. Principles and practice in area-wide integrated pest management. Dordrecht, The Netherlands: Springer.

Enserink, M. (2010). GM mosquito trial strains ties in Gates-funded project. Science, 330, 1030-1031. 
Nielsen, A. L., \& Burrus, R. G. (2010). The use of genetically modified organisms in entomology. American Entomologist, $56,106-114$.

Popovici, J., Moreira, L. A., Poinsignon, A., Iturbe-Ormaetxe, I., McNaughton, D., \& O'Neill, S. L. (2010). Assessing key safety concerns of a Wolbachia-based strategy to control dengue transmission by Aedes mosquitoes. Memórias do Instituto Oswaldo Cruz, Rio de Janeiro, 105, 957964.

\section{Further reading}

Anonymous. (2000). Cartagena protocol on biosafety to the convention on biological diversity. Secretariat of the Convention on Biological Diversity, Montreal, Canada.

Center for Disease Control and Prevention. Atlanta, GA, USA. http://www.cdc.gov/mmwr/preview/mmwrhtml/ mm5919a1.htm

Lara, L. E. Z., \& Lavery, J. V. (2010). Achieving precaution through effective community engagement in research with genetically modified mosquitoes. Asia Pacific Journal of Molecular Biology and Biotechnology, 18, 247-250.

Patil, P. B. M. S., Alam, P., Ghimire, R., Lacroix, P. H. D., Kusumawathie, R., Chowdhury, S., et al. (2010). Letter to the Editor: discussion on the proposed hypothetical risks in relation to open field release of a self-limiting transgenic Aedes aegypti mosquito strain to combat dengue. Asia Pacific Journal of Molecular Biology and Biotechnology, 18, 241-246.

Roberts, J. M. (2011). How western environmental policies are stunting economic growth in developing countries. The Heritage Foundation. http://report.heritage.org/bg2509.

Tan, S. C. (2010). Malaysia to release GM mosquitoes into the wild. Science and Development Network. http://www.scidev. net/en/news/malaysia-to-release-gm-mosquitoes-into-thewild.html.

Vreysen, M. J. B., Robinson, A. S., \& Hendrichs, J. (Eds.). (2007). Area-wide control of insect pests. From research to field implementation. Dordrecht, The Netherlands: Springer.

Wilson, E. K. (2011). Bruce Ames, developer of test for carcinogens now champions micronutrients for preventing disease. Chemical \& Engineering News, 14, 38. 